\title{
comportement aux séismes des tunnels et des ouvrages souterrains dans les sols*
}

\author{
behaviour of tunnels and underground structures \\ to earthquakes
}

\author{
Marc PANET \\ Président de SIMECSOL **
}

\section{Résumé}

Les tunnels et les ouvrages souterrains résistent en général mieux aux séismes que les structures de surface. L'amplitude des mouvements et des accélérations sont plus faibles en profondeur qu'en surface. Dans la plupart des cas on peut analyser les sollicitations des ouvrages souterrains en négligeant l'interaction sol-structure et les effets d'inertie. On suppose alors que le champ de déplacement imposé à la structure est la même qu'en champ libre. Dans le cas de structures rigides dans les sols mous, il est nécessaire de faire appel à des modèles numériques prenant en compte l'interaction sol-structure et les effets d'inertie.

\begin{abstract}
Tunne/s and underground structures exhibit a better resistance to earthquakes than surface structures. The main difficulties occur at soil discontinuities. The amplitude of motions and accelerations are smaller at depth than at the surface. In most cases, the seismic actions on underground works may be analysed by neglecting the soil-structure interaction and inertia forces. Then it is assumed that the displacement field assigned to the structure is the same as in the free field. For rigid structures excavated in soft soils, one must used numerical models taking into account the soil-structure interaction and inertia forces.
\end{abstract}

\footnotetext{
- «Communications aux journées communes C.F.M.S./A.F.P.S. des 18 et 19 novembre 1986 sur le thème : fondations, propriétés des sols et impératifs sismiques.

*115, rue Saint-Dominique - 75007 Paris.
} 
Les ouvrages souterrains (tunnels, métros, galeries d'adduction d'eau et d'assainissement, canalisations, ...) se comportent généralement bien sous les sollicitations de type sismique; pour un séisme d'intensité donnée, les désordres des ouvrages souterrains sont beaucoup plus faibles que les dommages des ouvrages construits à la surface du sol.

L'étude statistique la plus complète sur les dommages causés aux tunnels par les séismes a été réalisée par DOWDING et ROZEN (2). Elle porte essentiellement sur des ouvrages excavés dans les massifs rocheux et a trait à des tunnels ferroviaires ou des galeries hydrauliques dont l'ouverture varie entre $3 \mathrm{~m}$ et $6 \mathrm{~m}$, qui ont subi des tremblements de terre dont la magnitude varie de 5,8 à 8,3 et dont le foyer est situé entre 15 et $20 \mathrm{~km}$ de profondeur. L'importance des désordres est mise en relation avec l'accélération maximale en surface $\mathrm{a}_{0}$, ou avec la vitesse de vibration maximale en surface $v_{0}$. Il apparaît les règles empiriques suivantes (fig. 1) :

- si $\mathrm{a}_{0}<0,19 \mathrm{~g}$ ou $v_{0}<20 \mathrm{~cm} / \mathrm{s}$, aucun désordre n'est observé,

- si $\mathrm{a}_{0}>0,5 \mathrm{~g}$ ou $\mathrm{v}_{0}>90 \mathrm{~cm} / \mathrm{s}$, des désordres sérieux sont à craindre; on peut remarquer que ces seuils correspondent à des séismes très destructeurs pour les ouvrages superficiels.

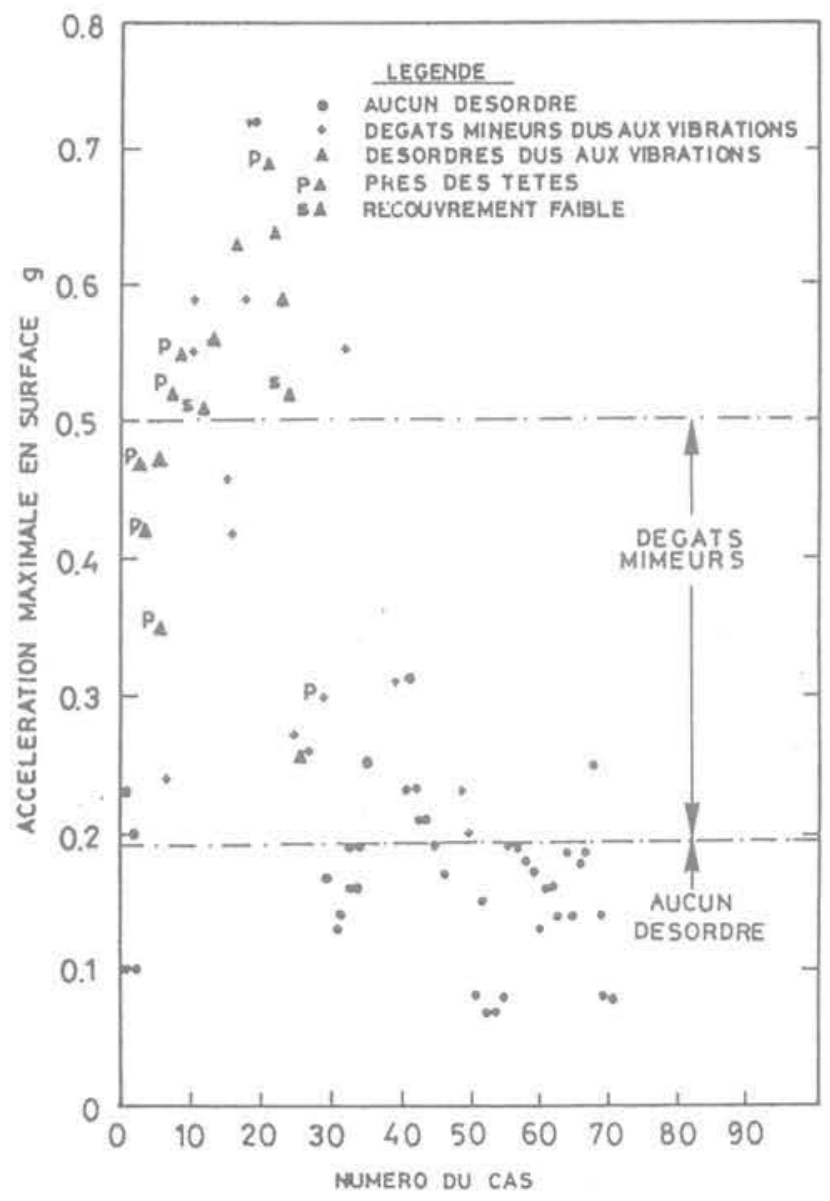

Fig. 1. - Effets des tremblements de terre sur les tunnels creusés dans les massifs rocheux (d'après DOWDING et ROZEN (2))
Les raisons essentielles de ce bon comportement aux séismes des ouvrages souterrains sont essentiellement les suivantes :

- les déplacements et les accélérations sont plus faibles en profondeur qu'à la surface du sol,

- il n'y a pas d'amplification des déplacements dans la structure souterraine par rapport aux déplacements du sol; on peut le plus souvent négliger les forces d'inertie de la structure.

En fait les principaux désordres subis par les ouvrages souterrains résultent de discontinuités dans le comportement des terrains encaissants telles que :

a) les zones de transition entre un sol mou et un sol raide ou du rocher;

b) les failles actives qui peuvent rejouer en cours de séisme et constituent une discontinuité cinématique. On cite souvent le cas du tunnel de Wright qui, lors du tremblement de terre de San Francisco en 1906 (avec une magnitude de 8,3 ), a été cisaillé au passage d'une faille avec un rejet de $13,70 \mathrm{~m}$;

c) les glissements de terrain dans les zones de tête;

d) les phénomènes de liquéfaction qui se produisent dans les zones de sols lâches et saturés.

Ce sont ces circonstances particulières qui sont à l'origine des désordres les plus graves subis par les ouvrages souterrains en cas de séismes. Ils ont été traités par ailleurs et nous ne les examinerons pas ici. Nous limiterons cette étude au comportement d'une galerie linéaire située dans un sol homogène dans lequel la continuité des déplacements est maintenue au cours du séisme. On considère des ondes sinusoïdales de la forme :

$$
u=\max (u) \sin 2 \pi \frac{x}{V T}
$$

$\mathrm{V}$, vitesse de propagation.

T, période.

\section{LES MOUVEMENTS SISMIQUES DANS LE SOUS-SOL}

L'amplitude des mouvements sismiques est plus faible en profondeur qu'en surface. La présence d'une couche de sol meuble au-dessus d'un substratum plus rigide a pour effet d'amplifier fortement en surface les vibrations pour des fréquences caractéristiques qui dépendent des caractéristiques de déformabilité du sol et de son épaisseur.

A titre d'exemple, la figure 2 montre pour un site alluvial les spectres de réponse en accélération à la surface du sol et à deux profondeurs différentes, $12 \mathrm{~m}$ et 40 m, du séisme de Salinas du 10 mars 1972.

Rappelons que pour un sol homogène d'épaisseur $\mathrm{H}_{c}$ au-dessus d'un substratum plus rigide, la fréquence propre fondamentale des ondes $\mathrm{SH}, \mathrm{N}_{1}$, est donnée 
pour un comportement élastique par :

$$
\mathrm{N}_{1}=\frac{\mathrm{V}_{\mathrm{S}}}{4 \mathrm{H}_{c}}
$$

où $\mathrm{V}_{s}$ est la vitesse de propagation des ondes $\mathrm{SH}$ dans le sol.
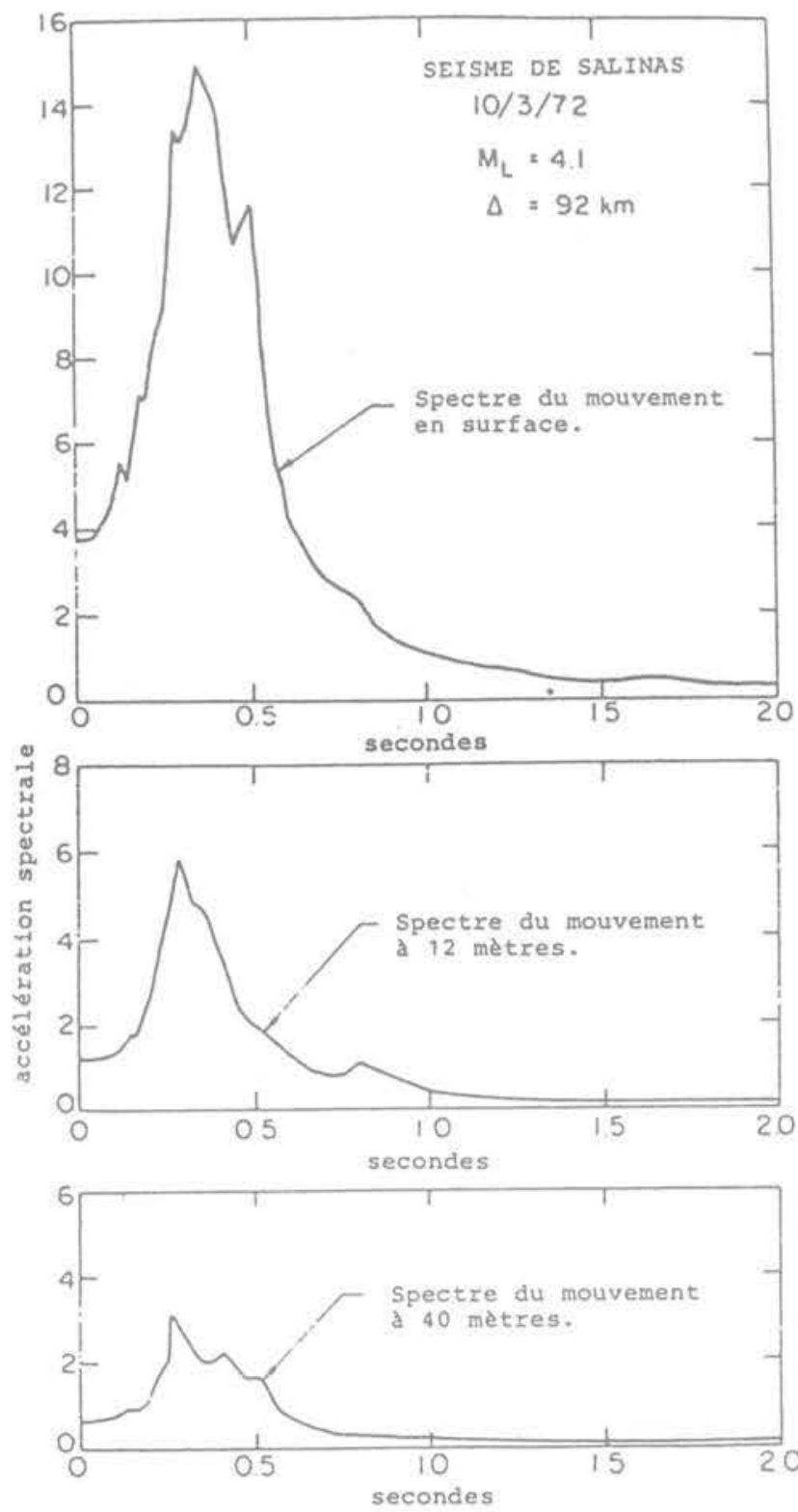

Fig. 2. - Exemple d'amplification du spectre de réponse en accélération due aux couches superficielles. Le site comprend $11 \mathrm{~m}$ de vase en surface, puis des alluvions (d'après SEED et LYSMER).

Pour une onde sinusoïdale de fréquence $\mathrm{N}_{1}$ se propageant verticalement, le profil des déplacements horizontaux est (fig. 3) :

$$
\mathrm{u}(\mathrm{z})=\mathrm{u}_{0} \cos \frac{\pi}{2} \frac{\mathrm{z}}{\mathrm{H}_{c}}
$$

$\mathrm{u}_{0}$ étant le déplacement en surface.

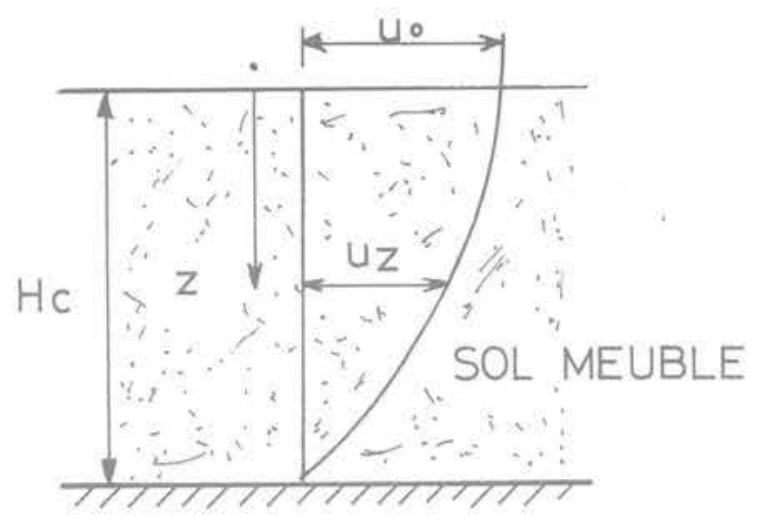

\section{SUBSTRATUM RIGIDE}

Fig. 3. - Profil en fonction de la profondeur des déplacements horizontaux.

Ainsi pour $\mathrm{H}_{\mathrm{c}}=50 \mathrm{~m}$ et $\mathrm{V}_{\mathrm{s}}=100 \mathrm{~m} / \mathrm{s}$, on trouve $\mathrm{N}_{1}=0,5$ et pour $\mathrm{z}=25 \mathrm{~m}$

$$
\mathrm{u}(25)=0,707 \mathrm{u}_{0}
$$

Les autres fréquences caractéristiques sont données par

$$
N_{n}=\frac{2 n-1}{4} \frac{V_{s}}{H_{c}}(n>1)
$$

En fait l'existence d'un amortissement propre du sol, d'origine visqueuse ou liée à l'hystérésis des lois de comportement, limite le nombre des modes de vibration; seuls les deux premiers modes ont un intérêt pratique.

Lorsque le sol est hétérogène, les modèles les plus simples pour étudier le profil des amplitudes connaissant le spectre d'accélération en surface ou au niveau de l'interface substratum rigide-sol, sont des modèles unidimensionnels linéaires équivalents dans lesquels les couches du sol sont représentées par des systèmes viscoélastiques en série excitées par une onde de cisaillement se propageant verticalement. Dans certains cas, il ne faut pas négliger les effets liés à la topographie, ou à la géométrie du substratum.

\section{LES SOLLICITATIONS SISMIQUES DES OUVRAGES SOUTERRAINS}

Pour l'étude des sollicitations d'un tunnel à axe horizontal situé à une profondeur $\mathrm{H}$ dans une couche de sol d'épaisseur $\mathrm{H}_{\mathrm{c}}$, on peut distinguer (fig. 4) :

- les déplacements dans le plan horizontal oxy qui créent des déformations axiales et des sollicitations de flexion :

- les déplacements dans le plan vertical oyz qui imposent une distorsion à la section transversale du tunnel.

Dans la très grande majorité des cas, on admet que les déplacements subis par l'ouvrage souterrain sont ceux du terrain encaissant en champ libre. L'interaction sol- 

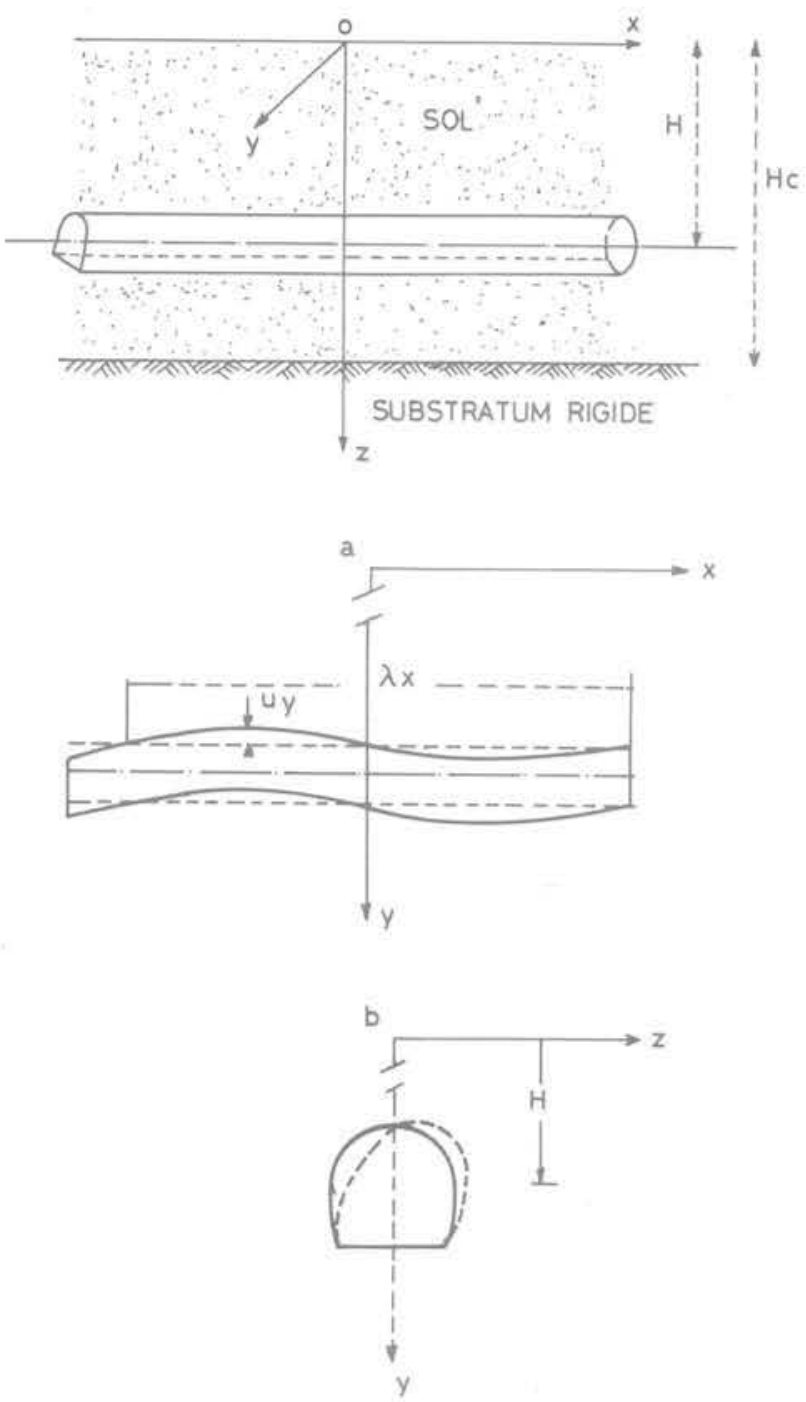

Fig. 4. - Déformations d'un tunnel à axe horizontal.

a) Flexion dans le plan horizontal oxy.

b) Distorsion dans le plan vertical oyz.

structure n'est pas prise en compte. Cette hypothèse demeure acceptable tant que les structures ont une rigidité faible vis-â-vis des terrains encaissants; elle devient discutable dans le cas des sols mous, dans lesquels les périodes de vibration sont peu élevées. Toutefois cette hypothèse va toujours dans le sens de la sécurité, puisque la rigidité de la structure a pour effet de s'opposer aux déplacements du sol et que par conséquent les déformations de la structure sont plus faibles.

La deuxième hypothèse, déjà mentionnée, consiste à négliger les effets d'inertie; elle n'est pas toujours justifiée comme le montrent les études sur modèles numériques. Il n'y a pas toujours continuité des déplacements à l'interface revêtement-sol, et des décollements peuvent se produire (5). Les conditions d'interface jouent un rôle important dans les modèles numériques. Si l'on ne suppose pas la continuité des déplacements à l'interface terrain-revêtement, et lorsqu'il y a localement dans la structure des masses concentrées, les effets d'inertie doivent être pris en compte.
Les analyses faites ci-dessous ne considèrent ni l'interaction sol-structure, ni les effets d'inertie, et sont par conséquent des analyses quasi statiques d'une structure à laquelle on impose un champ de déformation.

\section{ANALYSE DES SOLLICITATIONS LONGITUDINALES}

Dans le plan horizontal oxy on distingue :

- les déformations axiales parallèles à l'axe ox;

- les déformations de flexion dues aux composantes du déplacement perpendiculaires à ox.

3.1. Les déformations axiales soumettent le revétement d'un tunnel à des contraintes de compression et de traction qui se propagent le long du tunnel à la vitesse $V_{p}$, vitesse de propagation des ondes longitudinales. On peut évaluer la déformation axiale maximum, max $\varepsilon_{x}$, par la formule de Newmark correspondant à la déformation en champ libre

$$
\max \varepsilon_{x}=\frac{\max v_{x}}{v_{p}}
$$

où $v_{x}$ est la vitesse de vibration parallèlement à l'axe ox.

Le Comité technique japonais des joints parasismiques des pipe-lines souterrains propose une formule analogue

$$
\max \varepsilon_{x}=\frac{T_{1} \max a_{x}}{2 \pi V_{p}}
$$

où $\mathrm{a}_{\mathrm{x}}$ est l'accélération maximale suivant ox, et $\mathrm{T}_{1}$, la période fondamentale de vibration.

3.2. Une onde de cisaillement sinusoïdale de fréquence $\mathrm{N}$, se propageant à une vitesse $\mathrm{V}$, parallelement à l'axe ox, impose au tunnel d'épouser la forme d'une sinusoïde de longueur d'onde

$$
\lambda_{x}=\frac{V_{s}}{N}
$$

Les déformations de flexion créent alors des moments fléchissants $M$ et des efforts tranchants $T$ donnés respectivement par :

$$
\begin{aligned}
& M=E_{r} I_{r} \frac{d^{2} u_{y}(H)}{d x^{2}} \\
& T=E_{r} I_{f} \frac{d^{3} u_{y}(H)}{d x^{3}}
\end{aligned}
$$

où

$E_{\text {r }}$ est le module d'Young du revêtement;

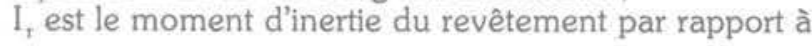
ox ;

$u_{y}(\mathrm{H})$ est le déplacement parallèlement à oy à la profondeur $\mathrm{H}$. 
Il en résulte que :

$$
\begin{aligned}
& M=-\frac{4 \pi^{2} E_{x} I_{y}}{\lambda_{x}^{2}} \max \left[u_{y}(H)\right] \sin 2 \pi \frac{x}{\lambda_{x}} \\
& T=-\frac{8 \pi^{3} E_{x} I_{x}}{\lambda^{3} x} \max \left[u_{y}(H)\right] \cos 2 \pi \frac{x}{\lambda_{x}}
\end{aligned}
$$

Le moment fléchissant et l'effort tranchant sont maximaux pour :

$$
x=\left(\frac{1}{4}+\frac{k}{2}\right) \lambda_{x}
$$

On a alors :

$$
|\max M|=\frac{4 \pi^{2} E_{\gamma} I_{\tau}}{2_{x}} \max \left[u_{y}(H)\right]
$$

et

$$
|\max T|=\frac{8 \pi^{3} \mathrm{E}_{\mathrm{t}} 1_{t}}{\lambda_{\mathrm{x}}^{3}} \max \left[\mathrm{u}_{\mathrm{y}}(\mathrm{H})\right]
$$

Soit encore :

$$
|\max M|=\frac{E_{t} I_{t}}{V_{s}{ }^{2}}\left[\max a_{y}(H)\right]
$$

et

$$
|\max T|=\frac{2 \pi \mathrm{NE}_{\mathrm{r}} \mathrm{I}_{\mathrm{r}}}{\mathrm{V}_{\mathrm{s}}{ }^{3}}\left[\max \mathrm{a}_{\mathrm{y}}(\mathrm{H})\right]
$$

où

$\mathrm{a}_{\mathrm{y}}(\mathrm{H})$ est l'accélération parallêle à oy à la profondeur $\mathrm{H}$.

Il apparait clairement que les sollicitations sont d'autant plus fortes que la vitesse de propagation des ondes de cisaillement est plus faible c'est-à-dire que le sol est plus mou.

A titre d'exemple, considérons un tunnel de section circulaire de $8 \mathrm{~m}$ de diamètre avec un revêtement en béton de $0,40 \mathrm{~m}$ d'épaisseur (module d'Young du béton $E_{t}=25000 \mathrm{MPa}$ ) dont l'axe est situé à $25 \mathrm{~m}$ de profondeur dans une couche de sol de $50 \mathrm{~cm}$ d'épaisseur au-dessus d'un substratum rigide. La vitesse de propagation des ondes de cisaillement $V_{s}$ est égale à $100 \mathrm{~m} / \mathrm{s}$ et $\mathrm{N}=0,5$. Les formules précédentes pour $\max \mathrm{a}_{y}(\mathrm{H})=0,2 \mathrm{~g}$ donnent :

$$
\begin{aligned}
& \max \mathrm{M}=914 \mathrm{MN} x \mathrm{~m} / \mathrm{n} \\
& \max \mathrm{T}=58 \mathrm{MN} / \mathrm{m}
\end{aligned}
$$

Comme le montre cet exemple, il est souvent nécessaire de limiter les moments fléchissants; on réalise alors des joints souples tous les quarts de longueur d'onde soit dans l'exemple précédent tous les $50 \mathrm{~m}$. La résistance du joint souple est alors calculée en admettant qu'il reprend un effort tranchant égal à l'effort de cisaillement engendré dans la section du sol correspondant à la section transversale du tunnel par le mouvement en champ libre.

3.3. Dans le cadre des études parasismiques pour le métro BART dans la baie de San Francisco qui a été en partie excavée dans des sols mous, KUESEL (4) a étudié les déformations du tunnel sous l'effet d'une onde de cisaillement se propageant suivant une direction faisant un angle $\psi$ avec l'axe du tunnel (fig. 5).

En supposant que la longueur d'onde $\lambda$ la plus défavorable est égale à six fois l'ouverture de l'excavation, la déformation maximale imposée à la structure souterraine est donnée par l'expression :

$$
\max \varepsilon=\frac{\pi(\max u(H))}{\lambda}\left[2 \sin \psi \cos \psi+\frac{\pi}{3} \cos ^{3} \psi\right]
$$

Fig. 5. - Déformation de flexion d'un tunnel sous l'effet d'une onde sinusoïdale oblique (d'après KUESEL).

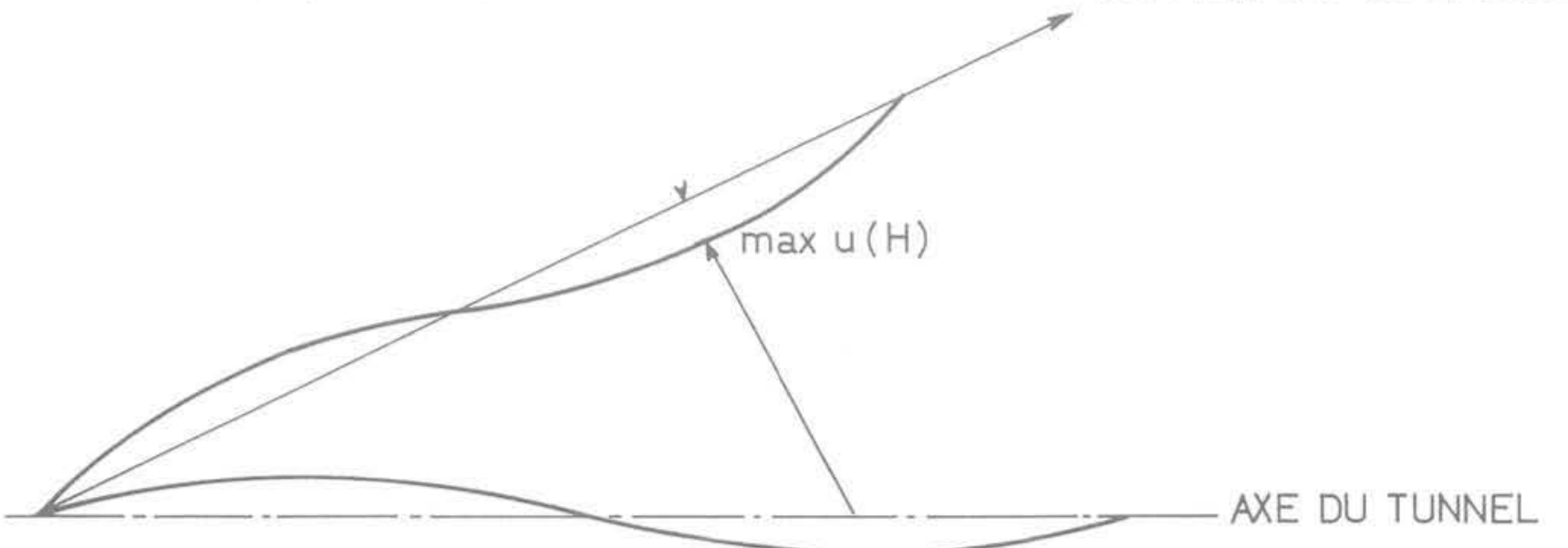


Elle est maximum pour $\psi=32^{\circ}$ et est alors égale à :

$$
\max \varepsilon=5,2 \frac{[\max u(\mathrm{H})]}{\lambda}
$$

KUESEL propose alors les règles suivantes :

- si max $\varepsilon<10^{-4}$, la structure demeure dans le domaine élastique;

- si max $\varepsilon>10^{-4}$, il convient de prévoir des joints pour absorber cette déformation.

\section{ANALYSE DES SOLLICITATIONS} TRANSVERSALES

4.1. Nous examinerons dans ce paragraphe le cas d'un ouvrage souterrain à axe horizontal situé à une profondeur $\mathrm{H}$ dans une couche de sol meuble d'épaisseur $\mathrm{H}_{c}$; les sollicitations dans le plan oyz sont dues à la propagation verticale d'une onde de cisaillement à partir d'un substratum rigide. Pour le mode fondamental $\mathrm{N}_{1}$, le profil du déplacement horizontal $\mathrm{u}_{\mathrm{y}}(\mathrm{z})$ est un quart de sinusoïde (fig. 3 ).

Ce déplacement impose à la section transversale du tunnel une distorsion (fig. 6).

La différence entre le déplacement horizontal de la clef de voute et le point le plus bas pour une section transversale circulaire de rayon $r$ est :

$$
2 \Delta u_{y}=\left(\frac{d u_{y}}{d z}\right) \times 2_{z}^{2}=H
$$

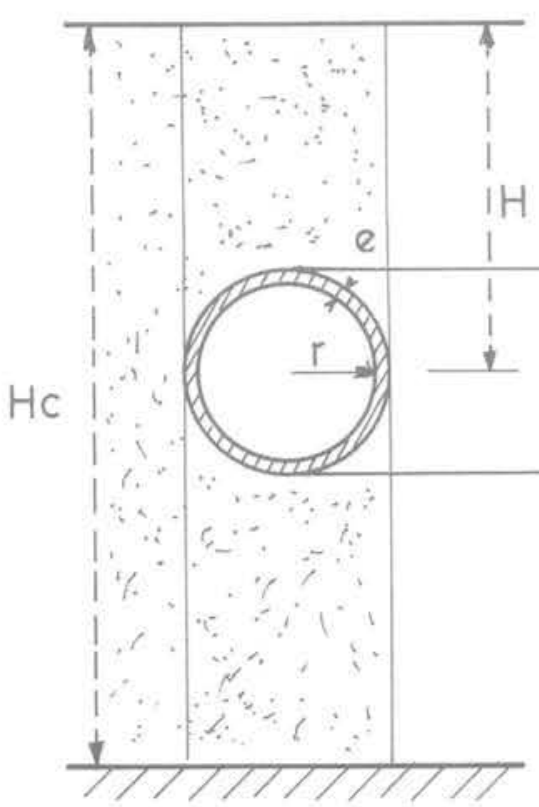

Soit la valeur maximale :

$$
\max \left(\Delta \mathrm{u}_{\mathrm{y}}\right)=\frac{2}{\pi} \frac{\mathrm{H}_{\mathrm{c}}}{\mathrm{V}_{\mathrm{s}}{ }^{2}} \mathrm{a}_{0} \mathrm{r} \sin \left(\frac{\pi}{2} \frac{\mathrm{H}}{\mathrm{H}_{\mathrm{c}}}\right)
$$

Les sollicitations du revêtement du tunnel dépendent de sa rigidité. Si le revêtement du tunnel est un anneau circulaire mince d'épaisseur e, la rigidité du revêtement peut être caractérisée par deux modules :

- un module de rigidité en compression

$$
\mathrm{K}_{\mathrm{sc}}=\frac{\mathrm{E}_{\mathrm{t}}}{\left(1-{\nu_{\mathrm{r}}}^{2}\right)} \frac{e}{\mathrm{r}}
$$

- un module de rigidité en flexion

$$
K_{s i}=9 \frac{E_{r}}{\left(1-v_{r}{ }^{2}\right)} \frac{I_{r}}{r^{3}}
$$

La contrainte radiale $\sigma_{r}$ et la contrainte de cisaillement $\tau_{\mathrm{r}^{\beta}}$ s'exerçant sur l'extrados du revêtement peuvent être déterminées à partir des équations différentielles (fig. 7) :

$$
\begin{gathered}
K_{s c}\left(\frac{d^{2} \nu_{r}}{d \theta^{2}}+\frac{d u_{r}}{d \theta}\right)=-r \tau_{r \theta} \\
K_{s c}\left(\frac{d v_{r}}{d \theta}+u_{r}\right)+\frac{1}{9} \\
K_{s i}\left(\frac{d^{4} u_{r}}{d \theta^{4}}+2 \frac{d^{2} u_{r}}{d \theta^{2}}+u_{r}\right)=r \sigma_{r}
\end{gathered}
$$

Fig. 6. - Distorsion imposée à la section transversale circulaire d'un tunnel. 


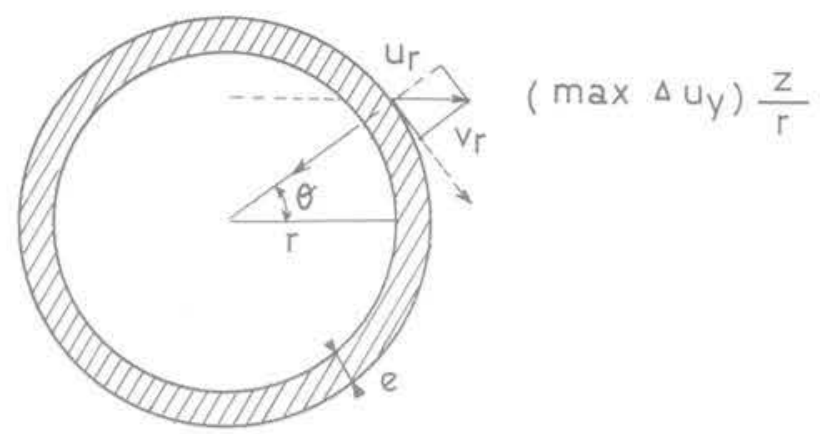

Fig. 7.

où les composantes radiale et tangentielle à l'extrados du revêtement sont données par :

$$
\begin{gathered}
u_{x}=-\frac{1}{2} \max \left(\Delta u_{y}\right) \sin 2 \theta \\
v_{x}=\max \left(\Delta u_{y}\right) \sin ^{2} \theta
\end{gathered}
$$

Il convient de noter que les contraintes de compression sont comptées positivement, et que le déplacement radial $u_{r}$ est positif lorsqu'il est centripète. On suppose également que sur la hauteur du tunnel la variation du déplacement horizontal $\mathrm{u}_{\mathrm{y}}$ est linéaire.

L'intégration des équations différentielles donne les expressions suivantes :

$$
\begin{gathered}
\sigma_{f}=\frac{1}{2} \frac{\max \left(\Delta u_{y}\right)}{r}\left(K_{s c}-K_{s f}\right) \sin 2 \theta \\
\tau_{r \theta}=-\frac{\max \left(\Delta u_{y}\right)}{r} K_{s c} \cos 2 \theta
\end{gathered}
$$

Elles permettent de déterminer l'effort normal $\mathrm{A}$ et le moment fléchissant $M$;

$$
\begin{aligned}
& A=\alpha r \sin 2 \theta \\
& M=\beta r^{2} \sin 2 \theta
\end{aligned}
$$

avec :

$$
\alpha=\frac{1}{2}\left(K_{s c}+\frac{1}{3} K_{s t}\right) \frac{\max \left(\Delta u_{y}\right)}{r}
$$

et :

$$
\beta=\frac{1}{6} K_{s i} \frac{\max \left(\Delta u_{y}\right)}{r}
$$

L'effort normal $A$ et le moment fléchissant $M$ sont maxima pour :

$$
\theta=\frac{\pi}{4}+\mathrm{k} \pi
$$

Si on reprend à titre d'exemple, le tunnel dans les conditions explicitées en 3.2. $(\mathrm{r}=4 \mathrm{~m} ; \mathrm{e}=0,4 \mathrm{~m}$;
$\left.\mathrm{E}_{\mathrm{t}}=25000 \mathrm{MPa} ; v_{\mathrm{t}}=0,15\right)$, les modules de rigidité sont :

$$
\begin{aligned}
& \mathrm{K}_{\mathrm{sc}}=2046 \mathrm{MPa} \\
& \mathrm{K}_{\mathrm{sf}}=16 \mathrm{MPa}
\end{aligned}
$$

d'où :

$\operatorname{Max} M=2,67 \frac{\max \left(\Delta u_{y}\right)}{r}$ exprimé en $M N \times m / m$ $\operatorname{Max} A=1025,5 \frac{\max \left(\Delta u_{y}\right)}{r}$ exprimé en $\mathrm{MN} / \mathrm{m}$

Les conditions $\mathrm{H}_{\mathrm{c}}=50 \mathrm{~m}, \mathrm{H}=25 \mathrm{~m}, \mathrm{~V}_{\mathrm{s}}=100 \mathrm{~m} / \mathrm{s}$ pour une accélération maximale en surface $\mathrm{a}_{0}=0,5 \mathrm{~g}$ conduisent à :

$$
\max \left(\Delta u_{y}\right)=1,1 \mathrm{~cm}
$$

d'où :

$$
\begin{aligned}
\operatorname{Max} M & =7,5 \mathrm{kN} \times \mathrm{m} / \mathrm{m} \\
\operatorname{Max} \mathrm{A} & =2820 \mathrm{kN} / \mathrm{m}
\end{aligned}
$$

Ces conditions sévères conduisent à des sollicitations maximales qui ne sont pas négligeables.

4.2. Pour l'étude du métro de la baie de San Francisco, KUESEL (4) a fait une étude sur modèle numérique pour étudier en champ libre la distorsion dans le plan vertical. Dans ce modèle, le module de déformation du sol croît proportionnellement avec la profondeur; le sol est soumis à sa base à un spectre d'accélération déduit des enregistrements du séisme d'El Centro en 1940, ajusté pour une accélération horizontale maximum de $0,33 \mathrm{~g}$ dans le substratum rocheux et de $0,5 \mathrm{~g}$ à la surface du sol. En se fondant sur les courbes de réponse, KUESEL propose une approximation linéaire donnant le déplacement horizontal maximum $\max \left[\mathrm{u}_{\mathrm{y}}\right]$ en fonction de la profondeur $\mathrm{z}$ (fig. 8)

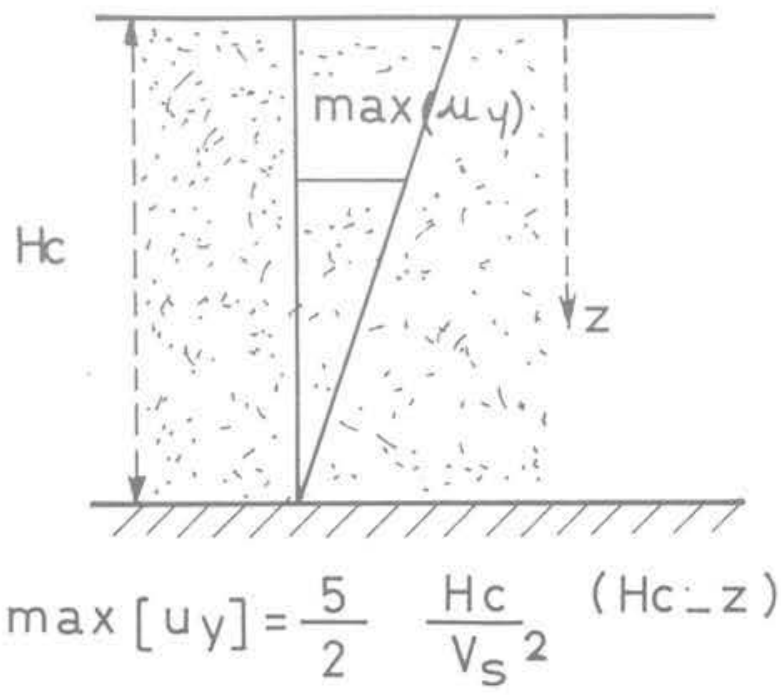

Fig. 8. 


$$
\max \Delta \mathrm{u}_{\mathrm{y}}=\frac{5}{2} \frac{\mathrm{H}_{\mathrm{c}}}{\mathrm{V}^{2}{ }_{\mathrm{s}}}\left(\mathrm{H}_{\mathrm{c}}-\mathrm{z}\right)
$$

où $u_{y}, H_{c}, z$ sont exprimés en pied, et $V_{s}$ en pied/seconde, $V_{s}$ étant la vitesse de propagation moyenne des ondes de cisaillement se propageant verticalement dans les sols.

On peut en déduire que, pour un tunnel horizontal de section circulaire à une profondeur $\mathrm{H}$, en utilisant les notations de 4.1. :

$$
\max \left(\Delta \mathrm{u}_{\mathrm{y}}\right)=\frac{5}{2} \frac{\mathrm{H}_{\mathrm{c}}}{\mathrm{V}_{\mathrm{s}}^{2}} \mathrm{r}
$$

Pour l'exemple numérique traité en 4.1 ., on trouve :

$$
\max \left(\Delta u_{y}\right)=1,5 \mathrm{~cm}
$$

valeur quelque peu supérieure à celle donnée en 4.1.

\section{CONCLUSIONS}

L'expérience montre que les ouvrages souterrains résistent en général bien aux séismes. Si on excepte les cas où les sols peuvent présenter de fortes discontinuités cinématiques, seuls les ouvrages situés dans des sols mous peuvent être soumis à des déformations trop élevées. Par des modèles analytiques simples, il est possible d'estimer les ordres de grandeur des sollicitations maximales dues aux séismes et de vérifier que l'ouvrage peut résister à ces sollicitations en plus des charges statiques permanentes. Il importe de ne pas perdre de vue que ces modèles simples ne tiennent pas compte de l'interaction sol-structure, ni des effets d'inertie. Lorsque les ouvrages ont une grande rigidité vis-à-vis des terrains encaissants, ces hypothèses ne sont souvent plus acceptables et il est préférable d'avoir alors recours à des modèles numériques.

Sur un plan pratique, il est le plus souvent préférable de réduire la rigidité des structures que de tenter d'accroître leur résistance, ce qui augmente généralement leur rigidité.

\section{BIBLIOGRAPHIE}

1. COUDERT J.F., PANET M., ROBERT J. (1986), Comportement aux séismes des tunnels et des ouvrages souterrains, Génie Parasismique, vol. VIII-8, pp. 817-831, Presses des Ponts et Chaussées, Paris.

2. DOWDING C.F., ROZEN A. (1978), Damage to rock tunnels from earthquake shaking, J. Geot. Eng., vol. CIV, GT2.

3. HALL W.J., NEWMARK N.M. (1977), Seismic design criteria for pipelines and facilities, Proc. Lifeline Earthquake Engineering Specialty Conference ASCE 1977, pp. 18-34.

4. KUESEL T.R. (1969), Earthquake design criteria for subways, J. Structural Division ASCE 95, ST 6. pp. 1213-1231.

5. PITILAKIS K. (1986), Analyse parasismique des tunnels et des pipe-lines, Méthodes de dimensionnement et expériences, Ier Colloque National de Génie Parasismique, Saint-Rémy-lès-Chevreuse, pp. 7/71-7/84. 